\title{
Patterns of Chest Wall Recurrence and Suggestions on the Clinical Target Volume of Breast Cancer: A Retrospective Analysis of I2I Postmastectomy Patients
}

Cancer Management and Research

\section{Lin-Wei Wang* \\ Li Li* \\ Hong-Yan Zhang \\ Yuan-Yuan Chen \\ Ya-Hua Zhong}

Department of Radiation and Medical Oncology, Zhongnan Hospital of Wuhan University, Hubei Key Laboratory of Tumor Biological Behaviors, Hubei Cancer Clinical Study Center, Wuhan, People's Republic of China

*These authors contributed equally to this work
Correspondence: Ya-Hua Zhong Email doctorzyh73@163.com
Purpose: Radiotherapy is a powerful strategy to prevent chest wall recurrence (CWR) of postmastectomy breast cancer $(\mathrm{BC})$. This retrospective study aims at analyzing patterns of CWR to explore the delineation of clinical target volume.

Patients and Methods: Detailed clinicopathological information of postmastectomy $\mathrm{BC}$ patients with CWR was collected from our single cancer center based on clear criteria. To describe recurrent positions more accurately, the chest wall was divided into three layers: skin layer (skin and subcutaneous tissues), pectoralis layer (pectoralis major and minor), and rib layer (rib and intercostal muscle). The frequency distribution of recurrence location and its association with clinical pathological factors were analyzed.

Results: A total of 121 postmastectomy BC with CWR were included in this study. The percentages of breast tumor located in the upper outer quadrant, upper inner quadrant, lower inner quadrant, lower outer quadrant, overlapping quadrant, and areola area were $31.0 \%$ (35/ 113), 26.5\% (30/113), 12.4\% (14/113), 5.3\% (6/113), 21.1\% (25/113), and 2.7\% (3/113), respectively. HER2-positive $\mathrm{BC}(51 / 113,45.1 \%)$ is the most common BC subtype. Analysis on the patterns of CWR showed that recurrences locating in the skin layer, pectoralis layer, rib layer, mixed layers, and incision periphery accounted for 58.6\% (68/116), 9.5\% (11/116), 1.7\% (2/116), 30.2\% (35/116), and 60.5\% (46/76), respectively. Rates of recurrences located in the skin and/or pectoralis layers for all BC patients, patients with concomitant distance metastasis, and patients without concomitant distance metastasis were $82.8 \%(96 / 116), 85.9 \%$ (49/57), and $81.0 \%$ (47/58), respectively.

Conclusion: For BC patients receiving mastectomy, skin, subcutaneous tissues, pectoralis, and area around incision have a high risk of recurrence, which should be paid more attention in chest wall radiotherapy.

Keywords: breast cancer, radiotherapy, chest wall recurrence, clinical target volume

\section{Introduction}

Breast cancer (BC) is the most common malignant cancer in females worldwide. ${ }^{1,2}$ Fortunately, the prognosis of $\mathrm{BC}$ has been improved greatly due to development in screening tools and comprehensive therapeutic strategies in the past decades. ${ }^{1-4}$ Operation, chemotherapy, radiotherapy, endocrinotherapy, targeted therapy, and immunotherapy play important roles in different stages of $\mathrm{BC}$. 5,6

Radiotherapy is a mainstay of $\mathrm{BC}$ treatment, widely used in $\mathrm{BC}$ patients receiving mastectomy and breast-conserving surgery, as well as in inoperable $\mathrm{BC}$ patients. ${ }^{7,8}$ 
Adjuvant radiotherapy post breast-conserving surgery reduces local recurrence and cancer-associated death. ${ }^{9}$ In patients treated with modified radical mastectomy, adjuvant radiotherapy can also improve local recurrence-free survival and overall survival. ${ }^{10,11}$ Therefore, radiotherapy following with surgery is recommended as an essential adjuvant therapy by various BC guidelines, especially for patients with large mass or/and lymph metastasis.

Chest wall recurrence (CWR) is the most common pattern of locoregional recurrence for mastectomy $\mathrm{BC}$ patients. ${ }^{12,13}$ Radiotherapy is a powerful locoregional strategy to reduce $\mathrm{BC}$ recurrence and deaths. With the rise of precise radiotherapy and development of radiotherapy technology, many concerns including less lung-heart dose, accurate target volume, small side effects from radiation therapy, and available patients have been raised in $\mathrm{BC}$ radiotherapy. ${ }^{14}$ For patients receiving mastectomy, the most common side effect of chest wall radiotherapy is radiotherapy-related pneumonia and cardiotoxicity. Therefore, an accurate delineation of clinical tumor volume (CTV) is the key to balancing the benefits and side effects of chest wall radiotherapy.

Target volume delineation guidelines for $\mathrm{BC}$ proposed by the Radiation Therapy Oncology Group (RTOG) ${ }^{15}$ and the European Society for Therapeutic Radiology and Oncology (ESTRO) $)^{16,17}$ are the two most commonly used guidelines. These two guidelines play huge roles in the implementation and development of $\mathrm{BC}$ radiotherapy. However, major disagreement about CTV definitions exists between two guidelines. Since there has not been a consensus on CTV definition, several retrospective studies tried to provide evidence to modify and optimize CTV delineations. ${ }^{13,18-20}$ These modified and optimal suggestions based on patterns of BC locoregional recurrences may contribute to formulating more reasonable and precise CTV delineations. However, few studies have focused on patterns of chest wall recurrence in detail.

Therefore, this retrospective single-center study aimed at exploring the recurrent CWR pattern of postmastectomy $\mathrm{BC}$ and its association with clinicopathological parameters. We also proposed a recommendation for chest wall CTV delineation based on our findings.

\section{Patients and Methods}

\section{Patients}

From Jan 1, 2013 to Jul 31, 2019, 121 postmastectomy BC patients with CWR diagnosed by pathological examination, imaging methods, or physical examination from our cancer center were included in this study. The inclusion criteria are: (1) patients receiving mastectomy; (2) age $\geq 18$ years; (3) no neoadjuvant therapy (including chemotherapy, radiotherapy, and endocrine therapy); (4) invasive $\mathrm{BC}$; (5) diagnosed as CWR for the first time. The exclusion criteria include male patients, metastasis at surgery, ductal carcinoma in situ, and bilateral BC. Detailed clinic-pathological information of those BC patients with CWR was recorded and analyzed. This study protocol was approved by the Institutional Ethics Committee of Zhongnan Hospital of Wuhan University and undertaken according to the ethical standards as laid down in the 1964 Declaration of Helsinki and its later amendments or comparable ethical standards.

\section{Definition of Chest Wall Recurrence for Breast Cancer}

Only recurrence of $\mathrm{BC}$ with mastectomy located in the chest wall scope was considered as CWR (or local recurrence). The scope of the chest wall in this study was defined as an irregular shape with four boundaries and guided by the contralateral breast. The upper boundary is the caudal border of the clavicle head, the lower boundary is the horizontal line of the lower margin of the contralateral breast, the inner boundary is the parasternal line and the outer boundary is the midaxillary line.

\section{Diagnosis of Chest Wall Recurrence}

CWR was defined as either of the following situations: (1) pathologic diagnosis: core needle biopsy or surgical excision that confirms recurrence from $\mathrm{BC}$; (2) imaging diagnosis: enhanced $\mathrm{CT}$ or enhanced MRI or PET/CT that shows new nodules/mass in chest wall; (3) physical examination: some small nodules of skin in chest wall diagnosed by physical examination (new and firm nodules, increase in size with time, exclude common benign skin diseases). To guarantee the accuracy of the diagnosis of tumor recurrence, the size of diseases diagnosed by imaging or physical examination should have changes (increase or shrinkage $\geq 5 \mathrm{~mm}$ ) in the subsequent anticancer therapies.

\section{Definition Chest Wall Layers and Recurrent Position}

To describe positions of recurrence more accurately and more precisely, the chest wall of the affected side was divided into three layers: the skin layer (including skin and subcutaneous tissue, green line area in Figure 1), the pectoralis layer (including pectoralis major and minor, the 


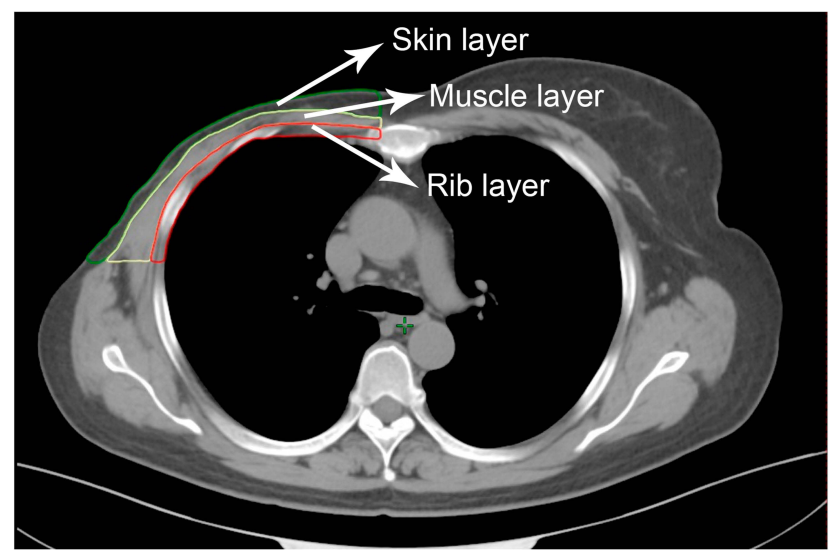

Figure I Example of chest wall layers. Green line area: skin layer including skin and subcutaneous tissue; yellow line area: pectoralis layer including pectoralis major and pectoralis minor; red line area: rib layer including rib and intercostal muscle.

yellow line area in Figure 1) and the rib layer (including rib and intercostal muscle, red line area in Figure 1).

\section{Statistical Analysis}

Data were recorded as categorical and continuous variables and analyzed by IBM Statistics, version 19.0 (IBM Corp, Armonk, NY, USA). Statistical methods consisted of frequency and chi-square $\left(\chi^{2}\right)$ analyses for categorical variables (Fisher's exact test was adopted if $\chi^{2}$ test was not applicable). Two-sided $P<0.05$ was judged as statistically significant.

\section{Results}

\section{Major Clinico-Pathological}

\section{Characteristics of BC Patients}

Major clinicopathological characteristics including patient age, T stage, $\mathrm{N}$ stage, concomitant metastasis at the time of recurrence, histological type, histological grade, hormone receptor (HR) status, human epidermal receptor-2 (HER2), and chest wall radiotherapy of $121 \mathrm{BC}$ patients with CWR are listed in Table 1. The median age of those patients was 49 years (range from 29 to 82 years). There were 61 cases $(61 / 121,50.4 \%)$ with concomitant metastasis at the time of CWR. The percentage of T2 and N0 patients ranked the highest, and accounted for 49.6\% (60/121) and 31.4\% (38/ 121), respectively. Invasive ductal carcinoma was the most common histology $(101 / 121,83.5 \%)$. Patients who were HR-positive and HER2 positive accounted for $47.9 \%$ (58/ $121)$ and $42.2 \%$ (51/121), respectively. The major clinicopathological information of $\mathrm{BC}$ patients is listed in supplementary files ( $\underline{\text { Table S1}})$.

\section{Quadrant Distribution of Breast Tumor}

There were 113 cases of breast tumor with definite quadrant distribution. The percentages of breast tumor located in the upper outer quadrant, upper inner quadrant, lower inner quadrant, lower outer quadrant, overlapping quadrant, and areola area were $31.0 \%$ (35/113), 26.5\% (30/113), 5.3\% (6/ $113), 12.4 \%$ (14/113), $22.1 \%(25 / 113)$, and $2.7 \%(3 / 113)$, respectively (Figure 2A).

\section{Diagnostic Methods of Chest Wall Recurrence}

Proportions of pathologic diagnosis, imaging diagnosis, and physical examination to diagnose CWR are shown in Figure 2B. For 121 cases of CWR BC patients, 76 cases (76/121, 62.8\%) were diagnosed by pathologic diagnosis (excisional biopsy: 59 cases, core needle biopsy: 17 cases), 28 cases $(28 / 121,23.1 \%)$ were diagnosed by imaging methods (enhanced CT: 19 cases, PET/CT: 7 cases, enhanced MRI: 2 cases), and 17 cases (17/121, 14.1\%) were diagnosed by physical examination.

\section{Molecular Subtype of Breast Cancer}

There were 8 cases lacking HR and/or HER2 information to confirm molecular subtype. For the other patients, HER2positive $\mathrm{BC}(51 / 113,45.1 \%)$ is the most common BC subtype. Thirty cases $(30 / 113,26.6 \%)$ were HER2-positive and HR-positive subtype, 21 cases $(21 / 113,18.6 \%)$ were HER2positive and HR-negative subtype, 37 cases $(37 / 113,32.7 \%)$ were triple-negative subtype and 25 cases $(25 / 113,22.1 \%)$ were luminal (HER2-negative) subtype (Figure 2C).

\section{Treatment Strategies for BC Patients}

All patients received modified radical mastectomy. A total of 102 cases finished the following adjuvant chemotherapy. For 51 cases who were HER 2 positive, only 3 cases $(3 / 51,5.9 \%)$ received 1 year of adjuvant anti-HER2 therapy. For 57 HRpositive patients, 19 patients (19/57, 33.3\%) did not follow endocrinotherapy advice, and 1 patient $(1 / 57,1.8 \%)$ had no information on endocrine therapy. In terms of adjuvant radiotherapy, 94 cases $(94 / 121,77.7 \%)$ did not receive radiotherapy, 26 cases $(26 / 121,21.5 \%)$ received postmastectomy radiotherapy, and 1 case $(1 / 121,0.8 \%)$ relapsed during radiotherapy.

\section{Interval Between Diagnosis and Chest Wall Recurrence}

For all patients, 49.6\% (60/121) CWR occurred in the first 2 years after the first diagnosis, $15.7 \%$ (19/121) CWR 
Table I Major Clinico-Pathological Characteristics of I2I BC Patients with CWR

\begin{tabular}{|c|c|}
\hline Items & n (\%) \\
\hline Median age (years) ${ }^{a}$ & $49(29-82)^{\mathrm{b}}$ \\
\hline \multicolumn{2}{|l|}{ T stage $(\mathrm{cm})$} \\
\hline $\mathrm{TI}$ & $8(6.6)$ \\
\hline $\mathrm{T} 2$ & $61(50.4)$ \\
\hline T3 & $13(10.7)$ \\
\hline $\mathrm{T} 4$ & $20(16.5)$ \\
\hline No data & $19(15.7)$ \\
\hline \multicolumn{2}{|l|}{ N stage } \\
\hline No & $37(30.6)$ \\
\hline $\mathrm{NI}$ & $31(25.6)$ \\
\hline N2 & II (9.1) \\
\hline N3 & $32(26.4)$ \\
\hline No data & $10(8.3)$ \\
\hline \multicolumn{2}{|l|}{ Metastasis } \\
\hline Yes & $6 \mathrm{I}(50.4 \%)$ \\
\hline No & $60(49.6 \%)$ \\
\hline \multicolumn{2}{|l|}{ Histological type } \\
\hline Invasive ductal carcinoma & $101(83.5)$ \\
\hline Other invasive carcinoma & $14(11.6)$ \\
\hline No data & $6(4.9)$ \\
\hline \multicolumn{2}{|l|}{ Histological grade } \\
\hline I & $0(0)$ \\
\hline II & $49(40.5)$ \\
\hline III & $30(24.8)$ \\
\hline No data & $42(34.7)$ \\
\hline \multicolumn{2}{|l|}{ HR status ${ }^{a}$} \\
\hline Positive & $59(48.8)$ \\
\hline Negative & $58(47.9)$ \\
\hline No data & $4(3.3)$ \\
\hline \multicolumn{2}{|l|}{ HER2 $^{\text {b }}$} \\
\hline Positive & $51(42.2)$ \\
\hline Negative & $62(5 \mid .2)$ \\
\hline No data/uncertain & $8(6.6)$ \\
\hline \multicolumn{2}{|l|}{ Received therapy strategies } \\
\hline Chemotherapy & $96(79.3)$ \\
\hline Endocrine therapy & $39(32.2)$ \\
\hline Targeted therapy & $3(2.5)$ \\
\hline Radiotherapy & $27(22.3)$ \\
\hline
\end{tabular}

Notes: ${ }^{a}$ Onset age; ${ }^{b}$ Range of age.

Abbreviations: HR, hormone receptor; HER2, human epidermal receptor-2.

occurred between the 2 nd and 3rd years after the first diagnosis, and $17.4 \%(21 / 121)$ CWR occurred between the 3rd and 5th years after the first diagnosis (Figure 2D). For patients who relapsed within 2 years after diagnosis, $68.3 \%$ $(41 / 60)$ did not receive chest radiotherapy. Ratios of recurrence within 2 years after diagnosis for T1, T2, T3, and T4 were 25\% (2/8), 42.6\% (26/61), 53.8\% (7/13), and $90.0 \%$ (18/20), respectively. And ratios of recurrence within 2 years after diagnosis for patients with $\mathrm{N}$ stages of N0, N1, N2, and N3 were 37.8\% (14/37), 32.3\% (10/31), 72.7\% (8/ 11 ), and $81.3 \%$ (26/32), respectively. When T and/or N stages occurred later in patients, a shorter interval between surgery and chest wall recurrence was seen.

\section{Locations of Chest Wall Recurrence}

Recurrent locations of 116 cases $(116 / 121,95.9 \%)$ could be defined by imaging, surgery, and physical examination. Distributions of the CWR location for all patients are shown in Figure 3A. According to definitions of chest wall layers, 68 cases $(68 / 116,58.6 \%), 11$ cases (11/116, 9.5\%), and 2 cases ( $2 /$ $116,1.7 \%$ ) of CWR occurred in the skin, the pectoralis, and the rib layers, respectively. Additionally, 35 cases of recurrent tumor $(35 / 116,30.2 \%)$ were located in the mixed layers, including 17 cases $(17 / 116,14.7 \%)$ recurring in the skin and pectoralis layers, 10 cases $(10 / 116,8.6 \%)$ recurring in pectoralis and rib layers, and 8 cases $(8 / 116,6.9 \%)$ recurring in the skin, pectoralis, and rib layers. Distributions of CWR location with concomitant distance metastasis and without concomitant distance metastasis are shown in Figure 3B and C, respectively. The skin layer was still the most common site for both groups (patients with concomitant distance metastasis: 64.9\% [37/57]; patients without concomitant distance metastasis: 53.4\% [31/ $58])$. Recurrences in the skin and/or pectoralis layers accounted for $82.8 \%(96 / 116), 85.9 \%(49 / 57)$, and $81.0 \%$ (47/58) in all $\mathrm{BC}$ patients, patients with concomitant distance metastasis, and patients without concomitant distance metastasis, respectively.

We performed a subgroup analysis by comparing patients with chest wall radiotherapy versus those without chest wall radiotherapy. Clinical characteristics of CWR patients who had received chest wall radiotherapy and had not received chest wall radiotherapy are shown in supplementary files (Table S2). The skin layer was the most common site for recurrences in the chest wall radiotherapy group (73.1\%, 19/ 26) and no chest wall radiotherapy group (51.1\%, 48/94). Recurrences in the skin and/or pectoralis layers accounted for $84.6 \%(22 / 26)$ and $76.6 \%$ (72/94) in patients who had received chest wall radiotherapy and had not received chest wall radiotherapy, respectively. To further analyze the recurrent location of the chest wall, the conception of incision recurrence was introduced in this study. Incision recurrence was defined as recurrence in the $2 \mathrm{~cm}$ scope around the surgical incision. According to this definition, there were 77 
A

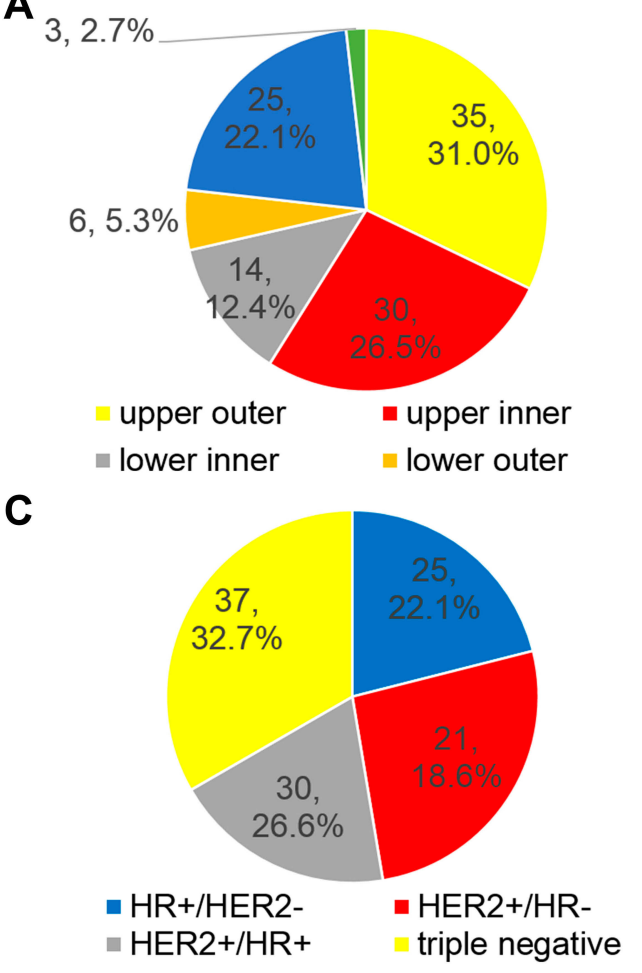

B

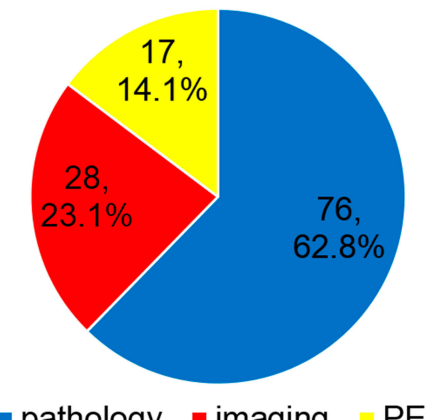

D

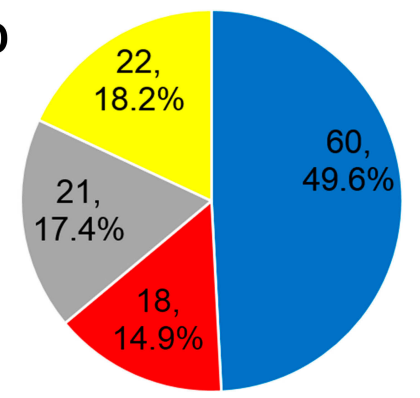

$\begin{array}{ll}-\leq 2 \text { years } & \quad>2 \text { and }<3 \text { years } \\ =\geq 3 \text { and }<5 \text { years } & \geq 5 \text { years }\end{array}$

Figure 2 Pie chart of proportions for BC. (A) Quadrant distribution of breast tumor location; (B) Diagnostic methods of CWR; (C) Molecular subtype of BC; (D) Interval time of surgery and CWR.

Abbreviations: PE, physical examination; HER2, human epidermal receptor-2; HR, hormone receptor.
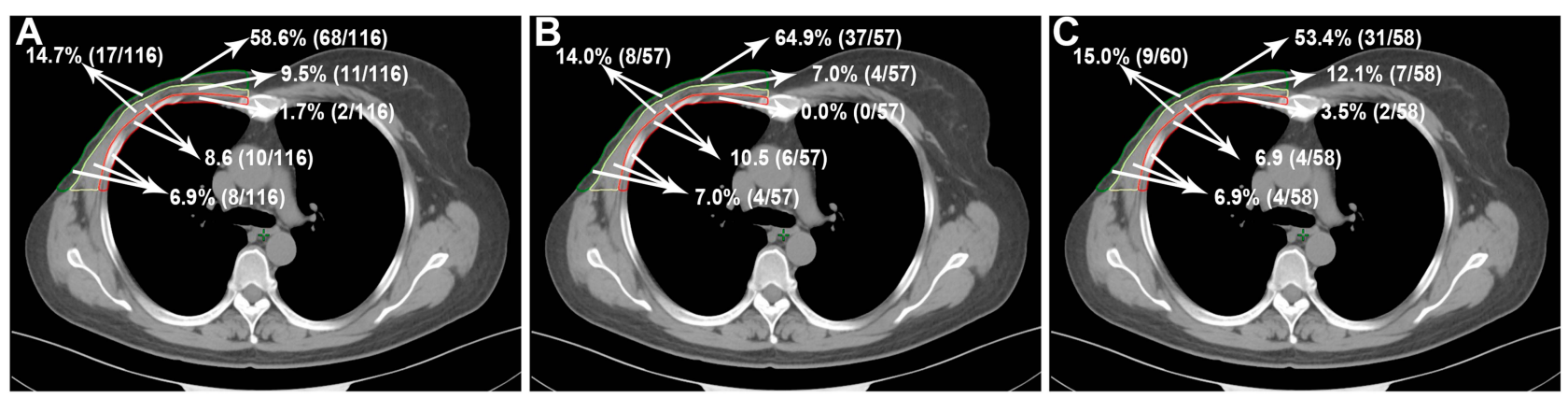

Figure 3 Distributions of location for CWR in all patients (A), patients with distance metastasis (B) and patients without metastasis (C).

cases with available information on incision recurrence. For those 77 patients, 47 cases $(61.0 \%, 47 / 77)$ were incision recurrence, and 30 cases $(38.2 \%, 30 / 77)$ were non-incision recurrence. Rates of incision recurrence in patients with concomitant metastasis and patients without concomitant metastasis were $47.6 \%$ (20/42) and 77.1\% (27/35), respectively.

\section{Distributions of Chest Wall Recurrence for Various $T$ and $N$ Stages}

Distributions of CWR for various $\mathrm{T}$ and $\mathrm{N}$ stages are shown in supplementary files (Table S3). Ratio of skin recurrence for T4 stage $(80 \%, 16 / 20)$ was higher than T1 $(50 \%, 4 / 8$, $P=0.112)$, T2 (52.3\%, 32/61, $P=0.030)$ and T3 (69.2\%, 9/13, $P=0.481)$ stages. Additionally, ratio of skin recurrence for N3 stage $(81.3 \%, 26 / 32)$ was higher than N2 stage $(72.7 \%$, $8 / 11, P=0.549)$, N1 stage $(48.4 \%, 15 / 31, P=0.006)$ and N0 stage $(40.5 \%, 15 / 37, P=0.001)$. When $\mathrm{T}$ or $\mathrm{N}$ stages occurred later in patients, there was a higher risk of skin recurrence.

\section{Typical Images of Chest Wall Recurrence}

Typical images of different CWR patterns are shown in Figure 4. According to the imaging diagnosis criterion, 
Figure $4 \mathrm{~A}-\mathrm{C}$ show tumor recurrence located in the skin layer. Figure 4D and $\mathrm{E}$ show tumor recurrence located in the pectoralis layer. Figure $4 \mathrm{~F}$ shows tumor recurrence in the rib layer. Figure 4G-I show tumor recurrence in mixed layers (Figure 4G: recurrence located in skin and pectoralis layers, Figure 4H: recurrence located in pectoralis and rib layers; Figure 4I: recurrence located in the skin, pectoralis, and rib layers).

\section{Examples of CTV Delineation for Chest Wall}

Examples of chest wall CTV delineation for postmastectomy patients in our cancer center are shown in Figure 5. CTV of the chest wall for $\mathrm{BC}$ was outlined on CT scan images for intensity-modulated radiotherapy. The surgical incision was labeled by a filiform marker, and surrounding boundaries of CTV were also labeled according to RTOG guidelines. For early $\mathrm{BC}$, the skin layer, pectoralis layer, and area around incision were included in CTV (Figure 5A). When the rib layer or deep pectoralis layer was invaded by the tumor (confirmed by pathology or imaging), the rib layer was included in CTV delineation (Figure 5B). The primary breast tumor location was a consideration for CTV delineation.

\section{Discussion}

Mastectomy used to be a typical surgical pattern and still plays an important role in $\mathrm{BC}$ surgical treatment. Along with the screening and early diagnosis of $\mathrm{BC}$, breast-conserving surgery has become the most common surgical pattern in many developed countries and areas. ${ }^{21}$ However, mastectomy still plays an important role in BC treatment, especially for advancedstage BC in developing countries. For patients receiving mastectomy, the chest wall is the most common site of locoregional recurrence. ${ }^{12,13}$ So, it is necessary to apply an effective strategy for preventing patients from tumor recurrence.

Radiotherapy is a powerful locoregional therapy strategy to protect $\mathrm{BC}$ patients from recurrence and to reduce $\mathrm{BC}$ mortality. There are two mainstream guidelines to describe in detail how to delineate CTV of BC: the RTOG guideline ${ }^{15}$ and the ESTRO guideline. ${ }^{16,17}$ For delineating CTV of the
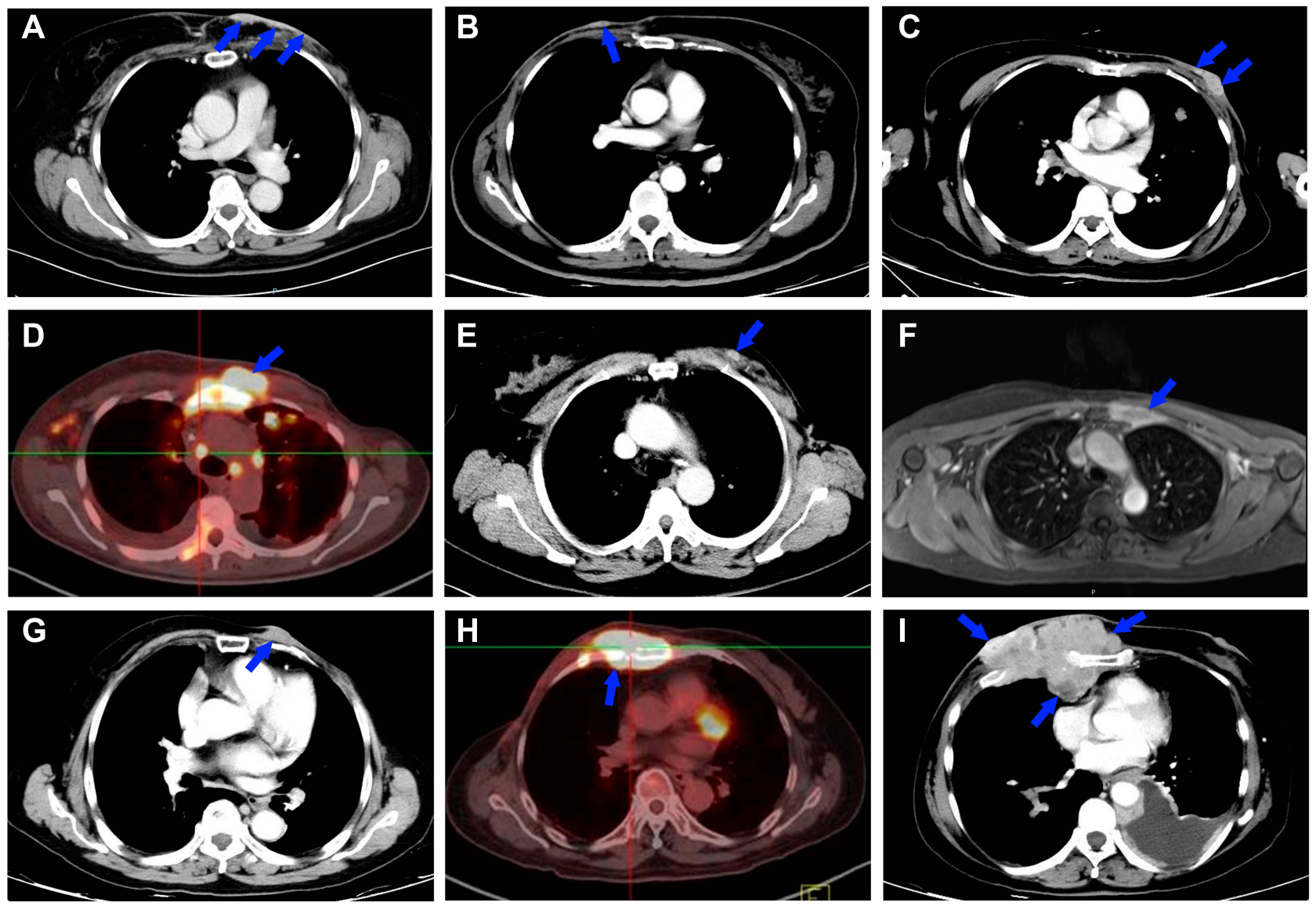

Figure 4 Typical imaging diagnosis examples of CWR for BC. (A-C) Recurrence in skin layer. (D and E) Recurrence in pectoralis layer. (F) Recurrence in rib layer. (G) Recurrence in skin and pectoralis layers; $(\mathbf{H})$ Recurrence in pectoralis and rib layers. (I) Recurrence in skin, pectoralis and rib layers. Blue arrows indicate recurrent chest wall tumor. 

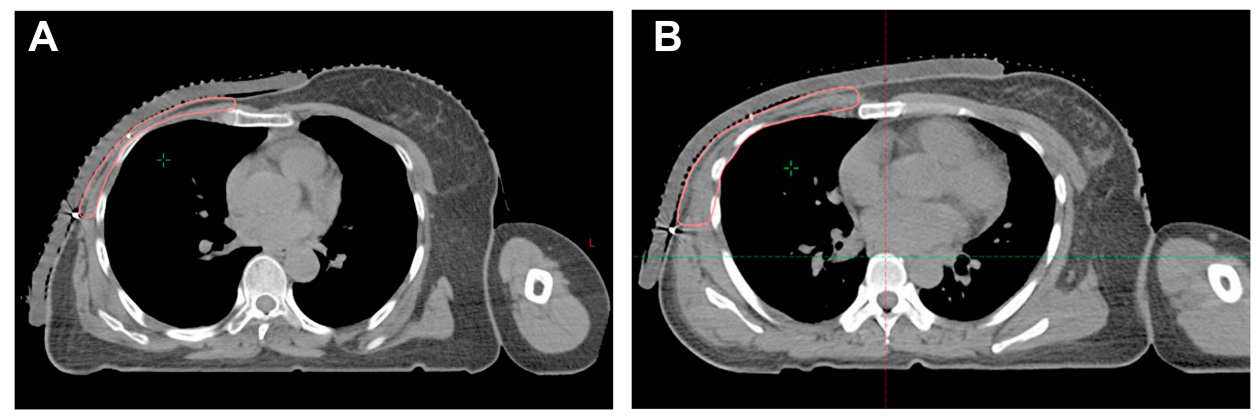

Figure 5 Examples of chest wall CTV delineation for patients undergoing mastectomy. (A) Skin layer, pectoralis layer and area around incision were included in CTV; (B) When rib layer or deep pectoralis layer was invaded by tumor (confirmed by pathology or imaging), the rib layer was included in CTV.

chest wall, there is major disagreement between those two guidelines. The RTOG guideline suggested that the chest wall should contain the skin layer, the pectoralis layer and the rib layer. ${ }^{15}$ However, the ESTRO guideline only recommended subcutaneous tissue $(5 \mathrm{~mm}$ under the skin surface to major pectoral muscle or costae, and intercostal muscles where no muscle) as CTV of the chest wall for early BC. ${ }^{16,17}$ Only large primary BC (pT3), locally advanced BC with nonpathological complete response to primary systemic therapy, and invasion of the major pectoral muscle and/or the chest wall pectoral muscle surface was considered as CTV in the ESTRO guideline. ${ }^{22}$

The two mainstream guidelines make a huge contribution to $\mathrm{BC}$ radiotherapy. However, in the definition of chest wall CTV the two guidelines have not reached an agreement. Given the obvious discrepancies, there have been many comments and comparative studies on those two guidelines. Based on retrospective recurrence data, several retrospective studies tried to present some proposals to optimize CTV delineations. $^{13,18-20}$ A comparative study reported by Chang et $\mathrm{al}^{13}$ showed that the ESTRO proposed CTV successfully covered the extent of the locoregional recurrence within the RTOG CTV in $96.4 \%$ of all early-stage breast cancer. And the result was also demonstrated in a subsequent multi-institutional validation study of the ESTRO consensus guideline. ${ }^{19}$ In terms of patients undergoing mastectomy, however, geographic misses of local recurrence for ESTRO CTV were higher than RTOG CTV. ${ }^{13}$ In a multi-institutional study, 24.1\% (7/29) postmastectomy patients had pectoralis muscle recurrence. ${ }^{19}$ Vargo et al identified 5 studies containing 278 patients with CWR patients who received postmastectomy from the database. ${ }^{20}$ Analysis of those $\mathrm{BC}$ patients showed $72-100 \%$ CWR occurred within skin and subcutaneous tissues anterior to the pectoralis musculature. ${ }^{23-27}$ The rest of chest recurrences were located in muscular areas within the pectoralis. None of the significant isolated recurrences were located in the intercostal muscles or ribs. $^{26,27}$ In view of the above results of studies, CTV of the chest wall for postmastectomy may be optimized rationally to increase local control ability and decrease complications.

Personalized and precision CTV delineation should take into account tumor stage, tumor location, molecular subtype, recurrence position, and other important clinical pathological parameters that might affect $\mathrm{BC}$ recurrence. Therefore, we performed this study aimed at exploring recurrent locations and clinical pathological parameters of postmastectomy BC patients with CWR. To guarantee the accuracy of diagnosis for tumor recurrence, pathological diagnosis was selected as the preferred diagnosis method in this study. In addition, recurrences diagnosed by imaging or physical examination should have changes (increase or shrinkage) in the subsequent anticancer therapies. Finally, 62.8\% (76/121), 23.1\% (28/121), and 14.1\% (9/121) patients were diagnosed as CWR by pathologic diagnosis, imaging methods, and physical examination, respectively.

Our study demonstrated skin layer (skin and subcutaneous tissues) was the most common recurrent location with a percentage of $58.6 \%$ (68/116). Furthermore, the skin layer was the most common site for subgroup analysis of chest wall radiotherapy or not and concomitant metastasis or not. And recurrences located in the skin and/or pectoralis layers accounted for $82.8 \%$ (96/116) BC patients. Only $2(2 / 116,1.7 \%)$ patients had isolated rib layer recurrence. Therefore, for the majority of $\mathrm{BC}$ patients with mastectomy, skin, and subcutaneous tissues were the most crucial CTV for chest wall radiotherapy due to the high risk of recurrence. To reduce radiotherapy-related damage (such as pneumonia and cardiotoxicity) and to perform precise radiotherapy, we suggested that chest wall CTV should not normally contain the rib layer unless the chest wall was very thin. Only when the rib layer or deep 
pectoralis layer were invaded by tumor (confirmed by pathology or imaging), should the rib layer be included in CTV. Subcutaneous lymphatic plexus should be irradiated for postmastectomy patients. ${ }^{22}$ And Shiau et $\mathrm{al}^{28}$ demonstrated that dose built up in about a $3 \mathrm{~mm}$ region beneath the skin and that the surface of the skin received only $52 \%$ of the prescribed dose. To prevent skin tissue from tumor recurrence, usually, the equivalent bolus may be considered to ensure an adequate dose in the skin.

It is reported that proportions of tumors located in the upper outer quadrant, upper inner quadrant, lower inner quadrant, and lower outer quadrant are $41.2-46.9 \%, 11 .-13.1 \%$, $5.6-5.8 \%$, and $8.2-8.5 \%$, respectively. ${ }^{29}$ The incidence of BC is proportional to the amount of breast tissue. ${ }^{30}$ In our study, the incidence of tumor in the upper outer quadrant is also the highest $(32.1 \%)$. However, the incidence of $\mathrm{BC}$ in the upper inner quadrant account for $26.8 \%$ in this study, twice what was reported (11.5-13.1\%). This may because breast tissue in the upper inner quadrant is thinner and less substantial, which allows the tumor to easily invade skin or muscle tissues to cause CWR. So, for patients with tumor located in the upper inner quadrant, chest wall radiotherapy may be recommended more positively.

The area around the operational incision was also a tissue with high recurrent risk whose recurrent rate was $61.0 \%$ (47/ 77). Therefore, during the delineation of target volume and implementation of radiotherapy, operational incision is an important area that should be paid more attention, to prevent missing target volume and keep enough radiation dose.

It has been demonstrated that anti-HER2 therapy is an essential treatment strategy for HER2-positive BC. ${ }^{31}$ In this study, the percentage of HER2-positive (HR \pm ) BC is $45.1 \%$ (51/113). However, only 3 cases have received 1-year adjuvant trastuzumab anti-HER2 therapy. It should be remembered that anti-HER2 therapy is also an effective strategy to prevent local recurrence. ${ }^{32,33}$ Analysis of distributions of CWR for various $\mathrm{T}$ and $\mathrm{N}$ stages showed the later $\mathrm{T}$ or $\mathrm{N}$ stages, the higher risk of skin recurrence. This may indicate radiotherapy is necessary to later $\mathrm{T}$ and $\mathrm{N}$ stage $\mathrm{BC}$.

Several limitations exist in this study. Firstly, this is a retrospective study with a small sample size, which may be influenced by many uncontrollable factors. So, the results of the study should be interpreted cautiously. Secondly, the association of recurrence patterns and $\mathrm{T} / \mathrm{N}$ stages is important to CTV delineation, but it was not analyzed due to the small sample size in this study. In the future, studies containing larger sample sizes can contribute to obtaining more reliable and abundant information on CWR and CTV delineation.

\section{Conclusion}

This study demonstrated that skin, subcutaneous tissues, pectoralis, and the area around the incision are areas with high recurrent risk for postmastectomy $\mathrm{BC}$ patients. More attention should be paid to those particular structures (skin layer and pectoralis layer) in delineating CTV of the chest wall. For patients with tumor located in the upper inner quadrant, chest wall radiotherapy should be strongly considered.

\section{Abbreviations}

$\mathrm{BC}$, breast cancer; CWR, chest wall recurrence; CTV, clinical tumor volume; ESTRO, European Society for Therapeutic Radiology and Oncology; HR, hormone receptor; HER2, human epidermal receptor-2; RTOG, Radiation Therapy Oncology Group.

\section{Data Sharing Statement}

The datasets used and/or analyzed during the current study are available from the corresponding author on reasonable request.

\section{Ethical Approval and Consent to Participate}

This study retrospectively collected the clinicopathological data of the patients, and did not collect the body fluids and tissue specimens of the patients. This study did not affect the treatment decision. All the research data were kept confidential and not used for commercial purposes. Exception to the requirement of informed consent was approved by the Institutional Ethics Committee of Zhongnan Hospital of Wuhan University (No. 2,017,057).

\section{Author Contributions}

All authors made substantial contributions to conception and design, acquisition of data, or analysis and interpretation of data; took part in drafting the article or revising it critically for important intellectual content; gave final approval of the version to be published; and agree to be accountable for all aspects of the work.

\section{Funding}

This work was supported by the Young Scientists Fund of National Natural Science Foundation (Grant Numbers 81701768 and 81702901) and Funding for Scientific and 
Technological Innovation of Zhongnan Hospital of Wuhan University (znpy2016003).

\section{Disclosure}

The authors declare no conflicts of interest.

\section{References}

1. Harbeck N, Gnant M. Breast cancer. Lancet. 2017;389 (10074):1134-1150. doi:10.1016/S0140-6736(16)31891-8

2. Bray F, Ferlay J, Soerjomataram I, et al. Global cancer statistics 2018: GLOBOCAN estimates of incidence and mortality worldwide for 36 cancers in 185 countries. CA Cancer J Clin. 2018;68 (6):394-424. doi:10.3322/caac.21492

3. Torre LA, Bray F, Siegel RL, et al. Global cancer statistics, 2012. $C A$ Cancer J Clin. 2015;65(2):87-108. doi:10.3322/caac.21262

4. Greenwood HI, Dodelzon K, Katzen JT. Impact of advancing technology on diagnosis and treatment of breast cancer. Surg Clin North Am. 2018;98(4):703-724. doi:10.1016/j.suc.2018.03.006

5. Odle TG. Precision medicine in breast cancer. Radiol Technol. 2017;88(4):401M-421M.

6. Schmid P, Adams S, Rugo HS, et al. Atezolizumab and nab-paclitaxel in advanced triple-negative breast cancer. $N$ Engl J Med. 2018;379 (22):2108-2121. doi:10.1056/NEJMoa1809615

7. Pinto JA, Saravia CH, Flores C, et al. Precision medicine for locally advanced breast cancer: frontiers and challenges in Latin America. Ecancermedicalscience. 2019;13:896. doi:103332/ecancer.2019.896

8. Lan XW, Wen G, He Z, et al. Comparison of long-term results between radiotherapy after breast-conserving surgery and postmastectomy radiotherapy in stage T1-2N1M0 breast cancer. Cancer Manag Res. 2019;11:6477-6487. doi:10.2147/CMAR.S209634

9. Early Breast Cancer Trialists' Collaborative Group. Effect of radiotherapy after breast-conserving surgery on 10-year recurrence and 15 -year breast cancer death: meta-analysis of individual patient data for 10,801 women in 17 randomised trials. Lancet. 2011;378 (9804):1707-1716. doi:10.1016/S0140-6736(11)61629-2

10. Ebctcg, McGale P, Taylor C, et al. Effect of radiotherapy after mastectomy and axillary surgery on 10-year recurrence and 20-year breast cancer mortality: meta-analysis of individual patient data for 8135 women in 22 randomised trials. Lancet. 2014;383 (9935):2127-2135. doi:10.1016/S0140-6736(14)60488-8

11. Poortmans P. Postmastectomy radiation in breast cancer with one to three involved lymph nodes: ending the debate. Lancet. 2014;383 (9935):2104-2106. doi:10.1016/S0140-6736(14)60192-6

12. Katz A, Strom EA, Buchholz TA, et al. Locoregional recurrence patterns after mastectomy and doxorubicin-based chemotherapy: implications for postoperative irradiation. J Clin Oncol. 2000;18 (15):2817-2827. doi:10.1200/JCO.2000.18.15.2817

13. Chang JS, Byun HK, Kim JW, et al. Three-dimensional analysis of patterns of locoregional recurrence after treatment in breast cancer patients: validation of the ESTRO consensus guideline on target volume. Radiother Oncol. 2017;122(1):24-29. doi:10.1016/j.radonc. 2016.10.016

14. Citrin DE, Longo DL. Recent developments in radiotherapy. $N$ Engl J Med. 2017;377(11):1065-1075. doi:10.1056/NEJMra1608986

15. White J, Tai A, Arthur D, et al. Breast cancer atlas for radiation therapy planning: consensus definitions. Available from: http://www. rtog.org/CoreLab/ContouringAtlases/BreastCancerAtlas.aspx. Accessed June 12, 2020.

16. Offersen BV, Boersma LJ, Kirkove C, et al. ESTRO consensus guideline on target volume delineation for elective radiation therapy of early stage breast cancer. Radiother Oncol. 2015;114(1):3-10. doi:10.1016/j.radonc.2014.11.030
17. Offersen BV, Boersma LJ, Kirkove C, et al. ESTRO consensus guideline on target volume delineation for elective radiation therapy of early stage breast cancer, version 1.1. Radiother Oncol. 2016;118 (1):205-208. doi:10.1016/j.radonc.2015.12.027

18. Jing H, Wang SL, Li J, et al. Mapping patterns of ipsilateral supraclavicular nodal metastases in breast cancer: rethinking the clinical target volume for high-risk patients. Int J Radiat Oncol Biol Phys. 2015;93(2):268-276. doi:10.1016/j.ijrobp.2015.08.022

19. Chang JS, Lee J, Chun M, et al. Mapping patterns of locoregional recurrence following contemporary treatment with radiation therapy for breast cancer: a multi-institutional validation study of the ESTRO consensus guideline on clinical target volume. Radiother Oncol. 2018;126(1):139-147. doi:10.1016/j.radonc.2017.09.031

20. Vargo JA, Beriwal S. RTOG chest wall contouring guidelines for post-mastectomy radiation therapy: is it evidence-based? Int $J$ Radiat Oncol Biol Phys. 2015;93(2):266-267. doi:10.1016/j.ijrobp.2015.03. 001

21. Ollila DW, Hwang ES, Brenin DR, et al. The changing paradigms for breast cancer surgery: performing fewer and less-invasive operations. Ann Surg Oncol. 2018;25(10):2807-2812. doi:10.1245/s10434-0186618-z

22. Kaidar-Person O, Vrou Offersen B, Hol S, et al. ESTRO ACROP consensus guideline for target volume delineation in the setting of postmastectomy radiation therapy after implant-based immediate reconstruction for early stage breast cancer. Radiother Oncol. 2019;137:159-166. doi:10.1016/j.radonc.2019.04.010

23. Slavin SA, Love SM, Goldwyn RM. Recurrent breast cancer following immediate reconstruction with myocutaneous flaps. Plast Reconstr Surg. 1994;93(6):1191-1204. doi:10.1097/00006534-199 405000-00013

24. Donegan WL, Perez-Mesa CM, Watson FR. A biostatistical study of locally recurrent breast carcinoma. Surg Gynecol Obstet. 1966;122 (3):529-540.

25. Gilliland MD, Barton RM, Copeland EM 3rd. The implications of local recurrence of breast cancer as the first site of therapeutic failure. Ann Surg. 1983;197(3):284-287. doi:10.1097/00000658-19830300000007

26. Langstein HN, Cheng MH, Singletary SE, et al. Breast cancer recurrence after immediate reconstruction: patterns and significance. Plast Reconstr Surg. 2003;111(2):712-720. doi:10.1097/01.PRS.00000414 41.42563 .95

27. Noone RB, Frazier TG, Noone GC, et al. Recurrence of breast carcinoma following immediate reconstruction: a 13-year review. Plast Reconstr Surg. 1994;93(1):96-106. doi:10.1097/00006534-199 401000-00014

28. Shiau AC, Chiu MC, Chen TH, et al. Surface and superficial dose dosimetric verification for postmastectomy radiotherapy. Med Dosim. 2012;37(4):417-424. doi:10.1016/j.meddos.2012.03.005

29. Bright CJ, Rea DW, Francis A, Feltbower RG. Comparison of quadrant-specific breast cancer incidence trends in the United States and England between 1975 and 2013. Cancer Epidemiol. 2016;44:186-194. doi:10.1016/j.canep.2016.08.019

30. Lee AH. Why is carcinoma of the breast more frequent in the upper outer quadrant? A case series based on needle core biopsy diagnoses. Breast. 2005;14(2):151-152. doi:10.1016/j.breast.2004.07.002

31. Loibl S, Gianni L. HER2-positive breast cancer. Lancet. 2017;389 (10087):2415-2429. doi:10.1016/S0140-6736(16)32417-5

32. He L, Wu Q, Xiong J, Su Z, Zhang B, Song Y. Do early HER2-overexpression breast cancer patients benefit from undergoing neoadjuvant trastuzumab and mastectomy? A meta-analysis. Cancer Manag Res. 2019;11:8043-8054. doi:10.2147/CMAR.S208319

33. Bellon JR, Guo H, Barry WT, et al. Local-regional recurrence in women with small node-negative, HER2-positive breast cancer: results from a prospective multi-institutional study (the APT trial). Breast Cancer Res Treat. 2019;176(2):303-310. doi:10.1007/s10549019-05238-4 


\section{Publish your work in this journal}

Cancer Management and Research is an international, peer-reviewed open access journal focusing on cancer research and the optimal use of preventative and integrated treatment interventions to achieve improved outcomes, enhanced survival and quality of life for the cancer patient.

The manuscript management system is completely online and includes a very quick and fair peer-review system, which is all easy to use. Visit http://www.dovepress.com/testimonials.php to read real quotes from published authors.

Submit your manuscript here: https://www.dovepress.com/cancer-management-and-research-journal 